\section{Kinderdermatologie kompakt}

D ie mehr als 40 Kapitel des vorliegenden Werkes stellen das gesamte Gebiet der pädiatrischen Dermatologie umfassend und übersichtlich dar. Sechs Jahre nach dem Ersterscheinen ist nun die zweite verbesserte und erweiterte Auflage neu auf dem Markt gekommen. Die Gliederung folgt einem bewährten Schema: Nach Embryologie und Neugeborenenzeit folgen Kapitel, die sich an der Morphe oder an der Ätiologie orientieren. Den Abschluss bilden Prinzipien der Therapie inklusive der operativen und lasergestützten Verfahren. Aufmachung und Konzeption sind überzeu- gend, Tabellen und Bilder lockern den Text auf, ohne ihn zu dominieren.

Als Autoren sind Dermatologen und Kinderärzte mit langjähriger Erfahrung in Klinik und Lehre versammelt, die in beiden Fachrichtungen zuhause sind. Neben Grundlagenwissen vermitteln die Verfasser auch Kenntnisse zu seltenen, aber wichtigen Krankheiten und Syndromen. Jedem Kapitel sind ausführliche Literaturstellen angefügt. Das Sachverzeichnis am Ende umfasst wertvolle 16 Seiten, könnte aber bei der nächsten Auflage noch eine zusätzliche Auflistung der Abbildungen und Tabellen enthalten.
Mit diesem Buch hält der Leser insgesamt ein modernes und gut ausgestattetes Buch über die Kinderdermatologie in Händen, das zum systematischen Nachschlagen und Durcharbeiten, aber auch einfach zum Durchstöbern einlädt.

Dr. Ulrich Mutschler, Hildesheim

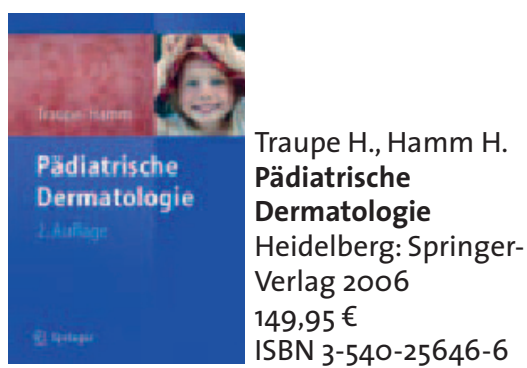

\section{Ekzem in allen Facetten}

S tattliche 15 Jahre nach der in vielen Arbeitsbereichen und Bibliotheken sehr gut eingeführten ersten Auflage des „Handbook of Atopic Eczema“ ist jetzt die zweite Auflage erschienen. Fast ist man versucht zu sagen: endlich ist sie da. Es handelt sich um ein englischsprachiges Vielautorenbuch. In 66 Kapiteln befassen sich international renommierte Kliniker und Forscher mit dem atopischen Ekzem und seinen verschie- denen Facetten: von der Historie über Staphylokokken, Eosinophile, dendritische Zellen und Mastzellen, Nahrungsmittel, Phosphodiesteraseinhibitoren bis hin zu Musiktherapie und psychobiologischen Aspekten. Entstanden ist ein praktisch keine Wünsche offen lassendes, sehr gut lesbares Buch. Kritisch anzumerken ist allenfalls, dass die klinischen Abbildungen von recht unterschiedlicher Qualität sind und die

Nomenklatur nicht einheitlich verwendet wird. Dem Werk ist eine weite Verbreitung zu wünschen. Es sollte in keiner Klinik oder Praxis fehlen.

Prof. Dr. Thomas Fuchs, Göttingen

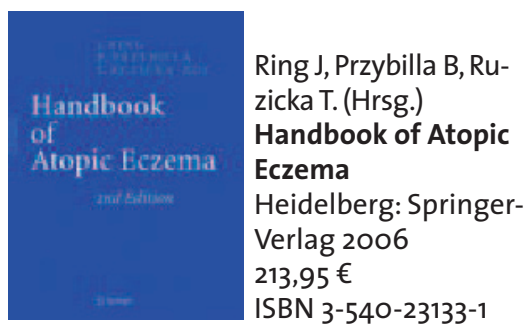

Das Buch ist insgesamt übersichtlich gegliedert und trotz wissenschaftlicher Diktion leicht zu lesen. Im Vergleich zu früheren Auflagen wurde auf eine bunte Bebilderung vollständig verzichtet - auch dadurch wird das Werk übersichtlicher und schlanker. Es sollte in keiner Schwerpunktpraxis fehlen.

Prof. Dr. Dietrich Berdel, Wesel ge vorliegende Buch aus dem Asthmazentrum Berchtesgaden aufzulösen. Es geht dabei nicht nur auf die theoretischen Erklärungsmodelle des Anstrengungsasthmas ein, sondern widmet sich auch den praktischen sportmedizinischen Aspekten. Als Zielgruppe werden damit nicht nur Wissenschaftler und Spezialisten, sondern auch Physiotherapeuten oder Asthmatrainer angesprochen.

Das Buch verschafft zunächst einen Überblick über verschiedene Entstehungshypothesen des kindlichen Asthmas. Es folgen ein Überblick über die Entstehungstheorien des Anstrengungsasthmas selbst sowie eine Darstellung der aktuellen Diagnostik und Therapie. Der sportmedizinische Teil befasst sich mit den erforderlichen Rahmenbedingungen der Sporttherapie mit asthmakranken Patienten und behandelt ausführlich die wichtigsten Sportarten. Das abschließende Kapitel widmet sich dem Thema „Asthma und Leistungssport" und spart auch die Dopingproblematik nicht aus.

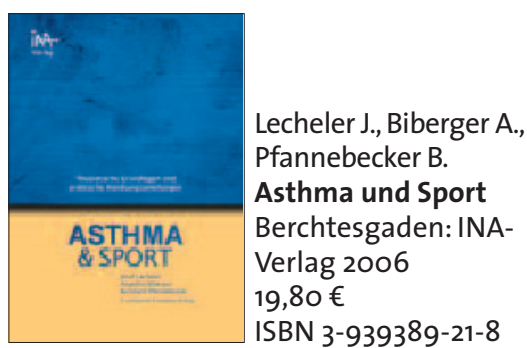

